\title{
Kinetics of the Enzymatic Degradation of Poly(vinyl acetate) in Solution
}

\author{
Sujay Chattopadhyay, Giridhar Madras \\ Department of Chemical Engineering, Indian Institute of Science, Bangalore, 560 012, India
}

Received 20 July 2002; accepted 26 October 2002

\begin{abstract}
The enzymatic degradability of poly(vinyl acetate) was investigated in toluene solutions at various temperatures with hog pancreas lipase. The polymer degraded by specific scission to yield oligomeric products with a molecular weight of 700 . Continuous distribution kinetics were used to determine the rate coefficients. The variation of the rate coefficients with the temperature indicated an optimum at $55^{\circ} \mathrm{C}$. The $p$-toluene sulfonic acid catalyzed degra-
\end{abstract}

dation of poly(vinyl acetate) was also investigated. The degradation mechanism was random chain scission, and the energy of activation for degradation was determined from the variation of the rate coefficients with the temperature. (C) 2003 Wiley Periodicals, Inc. J Appl Polym Sci 89: 2579-2582, 2003

Key words: degradation; enzymes; kinetics (polym.); high performance liquid chromatography (HPLC)

\section{INTRODUCTION}

Because of the environmental problems posed by conventional plastics, biodegradable polymers have gained considerable significance over the past 2 decades, particularly in the packaging industry. It has been estimated ${ }^{1}$ that nearly $50 \%$ of the annual world production of plastics will be used for packaging, of which $90 \%$ will end up as municipal waste. Several studies have investigated the synthesis of polymers based on cellulose or aliphatic polyester. Poly(lactic acid) (PLA) and poly( $\epsilon$-caprolactone) have been used extensively as biodegradable polymers, especially in biomedicine. ${ }^{2}$ Blends of biodegradable polymers and synthetic thermoplastics have been investigated. Thermoplastic poly(vinyl acetate) (PVAc) is blended with PLA to improve its tensile strength and other physical properties. It has been shown ${ }^{3}$ that increasing the PVAc content in the blend reduces the degradation. An understanding of the degradation behavior of PLA-PVAc blends requires a detailed investigation of the degradation kinetics of each polymer. We have chosen hog pancreas lipase (HPL) because it is cheap and commercially available.

To the best of our knowledge, we are unaware of any study that uses this lipase for the enzymatic degradation of polymers. Furthermore, this is also the first study of the enzyme-catalyzed degradation of PVAc at

Correspondence to G. Madras (giridhar@chemeng.iisc. ernet.in).

Contract grant sponsor: Indian Department of Science and Technology.

Journal of Applied Polymer Science, Vol. 89, 2579-2582 (2003) (C) 2003 Wiley Periodicals, Inc. various temperatures. The enzyme-catalyzed degradation refers to the side-chain hydrolysis of PVAc. This is compared with the kinetics of the $p$-toluene sulfonic acid (PTS) catalyzed degradation of PVAc at various temperatures. Continuous distribution kinetics are used to determine the rate coefficients from the time evolution of the molecular weight distribution (MWD).

\section{EXPERIMENTAL}

PVAc was synthesized by bulk polymerization and then precipitated with a nonsolvent to obtain a narrow distribution. The initial polymer was characterized by $\mathrm{NMR}$, and ester linkages $\left(-\mathrm{O}-\mathrm{C}(\mathrm{O})-\mathrm{CH}_{2}-\right)$ were found in the side chains of the polymer. The polymer used in the experiments had a number-average molecular weight $\left(M_{n}\right)$ of 210,000 and a polydispersity of 1.25. The polymer was dissolved in distilled toluene to prepare a $2 \mathrm{~kg} \mathrm{~m}^{-3}$ solution; $20 \mathrm{~cm}^{3}$ of this solution was placed in screw-cap cultured tubes kept in a constant-temperature bath. Depending on the reaction system, $0.01 \mathrm{~g}$ of HPL or PTS was added to each of the culture tubes, and the tubes were closed tightly. Samples of $0.5 \mathrm{~cm}^{3}$ of the solutions were taken at regular time intervals for subsequent analysis by gel permeation chromatography, as detailed in our previous work. ${ }^{4}$

\section{THEORETICAL MODEL}

It was observed experimentally that the enzymatic degradation of the polymer was by specific chain scission. Therefore, in the chromatograph, the intensity of the polymer peak gradually decreased, and peaks due 
to oligomers with a molecular weight (MW) of nearly 700 appeared. This indicated that the degradation of PVAc was specific. However, in the case of the acidcatalyzed degradation of PVAc, the MWD shifted to a lower MW without any specific products with an MW of 700, and this indicated that the scission of the polymer backbone was random. Therefore, the degradation of the polymer by enzymes is such that the ester bonds in the side chains are broken specifically to yield oligomers with acid and alcohol groups. There are many side branches in PVAc because chain transfer to the methyl groups in the acetate groups of PVAc takes place frequently during the polymerization of vinyl acetate. In the case of polymer degradation catalyzed by PTS, the mechanism is ionic. PTS initially attacks the ester side chains, and this results in the formation of a carbocation. The resulting polymeric carbocation undergoes $\beta$ scission, which results in a drop in the MW.

\section{Enzymatic degradation kinetics}

A generalized reaction, with a degradation rate coefficient $k_{s}$, in which a polymer with an MW of $x^{\prime}, \mathrm{P}\left(x^{\prime}\right)$, breaks down into a polymer with an MW of $x^{\prime}-x_{s^{\prime}}$ $\mathrm{P}\left(\mathrm{x}^{\prime}-x_{\mathrm{s}}\right)$, and an oligomer (specific product) with an MW of $x_{s}, \mathrm{Q}\left(x_{s}\right)$, can be written as follows: $:^{5}$

$$
\mathrm{P}\left(x^{\prime}\right) \rightarrow \mathrm{P}\left(x^{\prime}-x_{s}\right)+\mathrm{Q}\left(x_{s}\right)
$$

The MWDs of the chemical species $\mathrm{P}\left(x^{\prime}\right)$ and $\mathrm{Q}\left(x_{s}\right)$ are represented by $p\left(x^{\prime}, t\right)$ and $q\left(x_{s}, t\right)$, respectively. Therefore, at time $t, p\left(x^{\prime}, t\right) d x^{\prime}$ represents the molar concentration of the polymer with values of $x^{\prime}$ in the range of $x^{\prime}$ to $x^{\prime}+d x^{\prime}$. On the basis of this formulation, the time-dependent specific product concentration can be written as follows: ${ }^{5}$

$$
\partial q\left(x_{s}, t\right) / \partial t=\int_{x}^{\infty} k_{s} p\left(x^{\prime}, t\right) \delta\left(x^{\prime}-x_{s}\right) d x^{\prime}
$$

where the dirac $\delta$ function represents the scission of the specific product. Applying the moment operation, $q^{(n)}(t)=\int_{0}^{\infty} x^{n} q(x, t) d x$, to eq. (1) yields

$$
d q^{(n)} / d t=k_{s} p^{(0)} x_{s}{ }^{n}
$$

where $p^{(0)}$ represents the zero moment (molar concentration) of the polymer. Equation (A) indicates that the molar concentration of the polymer remains unchanged with time, that is, $p^{(0)}=p_{0}{ }^{(0)}$, the initial molar polymer concentration. Equation (2) can be solved to obtain the moments of the specific product. The first moment, $n=1$, corresponding to the mass concentration, of the specific product is

$$
d q^{(1)} / d t=k_{s} x_{s} p^{(0)}
$$

Integrating this equation with the initial condition, $q^{(1)}(t=0)=0$, yields

$$
q^{(1)}=k_{s} x_{s} p^{(0)} t
$$

Equation (4) depicts the variation of the mass concentration of the specific product with the reaction time.

\section{PTS degradation kinetics}

The degradation of PVAc catalyzed by PTS is by an ionic mechanism. It has been determined experimentally that the polymer is broken by random chain scission and that no specific products are formed. A generalized reaction, with a degradation rate coefficient $k_{r}$, in which $\mathrm{P}\left(x^{\prime}\right)$ breaks into two polymer fragments of $\mathrm{MW}=x^{\prime}-x$ and $\mathrm{MW}=x$ can be written as follows:

$$
\mathrm{P}\left(x^{\prime}\right) \rightarrow \mathrm{P}(x)+\mathrm{P}\left(x^{\prime}-x\right)
$$

The rate of change of the polymer MWD is

$$
\begin{aligned}
\partial p(x, t) / \partial t=- & k_{r}(x) p(x, t) \\
& +2 \int_{0}^{\infty} k_{r}(x) p\left(x^{\prime}, t\right) \Omega\left(x, x^{\prime}\right) d x^{\prime}
\end{aligned}
$$

$\Omega\left(x, x^{\prime}\right)$ denotes the scission stoichiometric kernel and is equal to $1 / x^{\prime}$ for random chain scission. It is assumed that the degradation rate coefficient depends linearly on the chain length, $k_{r}(x)=k_{r} x$. Applying the moment operation to eq. (5) yields

$$
d p^{(n)} / d t=-[(n-1) /(n+1)] k_{r} p^{(n+1)}
$$

For $n=0$, the zero moment corresponding to the molar concentration of the polymer is

$$
d p^{(0)} / d t=k_{r} p^{(1)}
$$

The first moment $(n=1)$, corresponding to the mass concentration of the polymer, is

$$
d p^{(1)} / d t=0
$$

Equation $(7 b)$ indicates that the mass of the polymer remains unchanged during the degradation process, $p^{(1)}=p_{0}{ }^{(1)}$. Integrating eq. (6) yields $p^{(0)}-p_{0}{ }^{(0)}$ $=k_{r} p_{0}{ }^{(1)} t$. Because $M_{n}$ is defined as $p^{(1)} / p^{(0)}$, this yields

$$
M_{n}{ }^{-1}-M_{n 0}{ }^{-1}=k_{r} t
$$




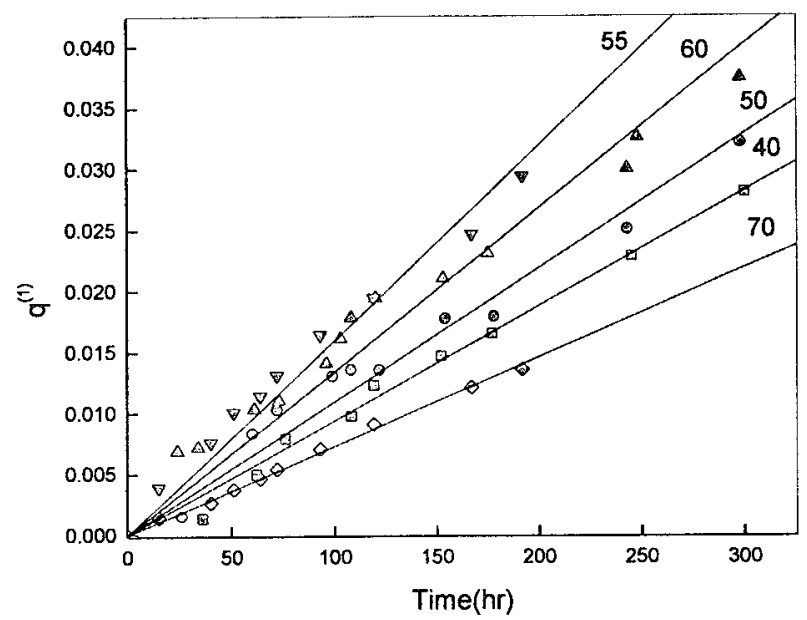

Figure 1 Variation of the specific product mass concentration, $q^{(1)}(\mathrm{g} / \mathrm{L})$, with the reaction time. The numbers on the line indicate the various temperatures $\left({ }^{\circ} \mathrm{C}\right)$.

Equation (8) shows the variation of $M_{n}$ with time for the PTS-catalyzed degradation of PVAc.

\section{RESULTS AND DISCUSSION}

We investigated the degradation of PVAc by catalyzing the degradation with an enzyme and PTS. For the enzymatic degradation of PVAc, eq. (4) indicates that the mass of the specific product increases linearly with time. The experimental data, shown in Figure 1, indicates that the proposed model can satisfactorily explain the observation within the experimental error. Triplicate experiments for several experiments indicated a variation of the oligomeric mass, $q^{(1)}$, of less than $1 \%$. Based on eq. (4), $k_{s}$ is determined from the slope of the lines in Figure 1. The variation of the rate coefficients with the temperature is shown in Figure 2, indicating an optimum temperature of $55^{\circ} \mathrm{C}$ for the

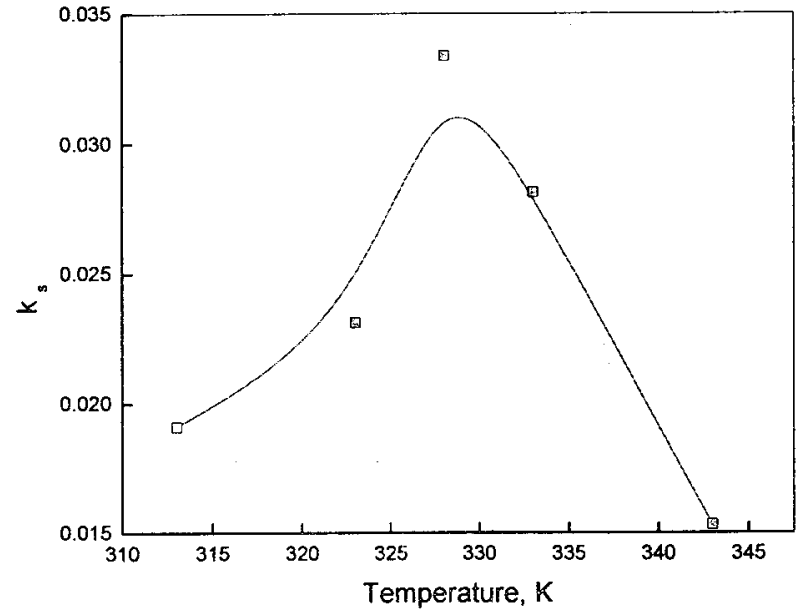

Figure 2 Variation of the degradation rate coefficient, $k_{s}$, for the enzymatic degradation of PVAc with the temperature, indicating an optimum temperature for the reaction.

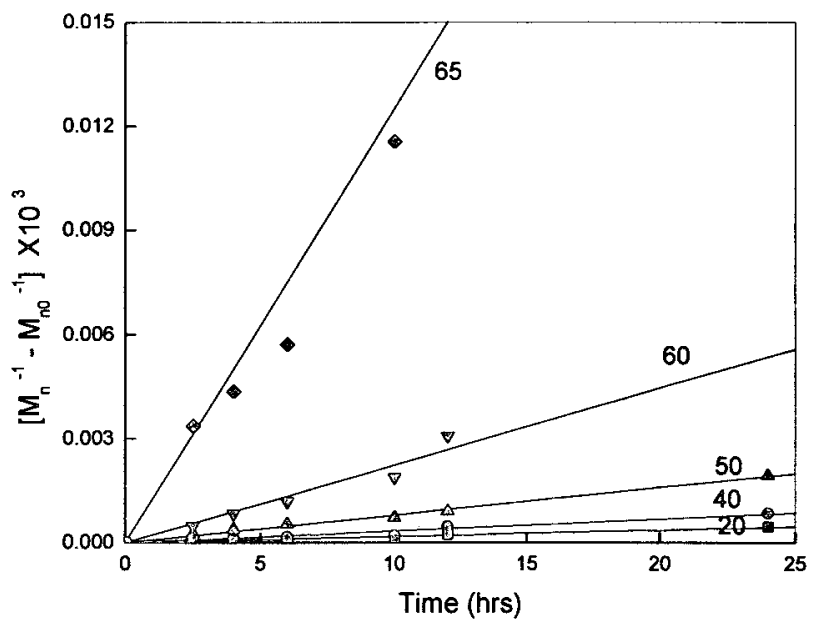

Figure 3 Plot of $\left(1 / M_{n}\right)-\left(1 / M_{n 0}\right)$ versus the reaction time for the acid-catalyzed degradation of PVAc at various temperatures. The numbers on the lines indicate the various temperatures $\left({ }^{\circ} \mathrm{C}\right)$.

enzymatic degradation of PVAc investigated in this study. This optimum temperature is typical for hydrolysis and esterification reactions catalyzed by this enzyme. ${ }^{6,7}$

The degradation of PVAc catalyzed by PTS occurs by random chain scission in the polymer backbone. Equation (8) implies that the variation of $M_{n}$ with time is linear, as shown in Figure 3. The variation of $k_{r}$, obtained from the slopes of the lines in Figure 3, with the temperature can be represented in an Arrhenius plot (Fig. 4). The energy of the activation, obtained from Figure 4 , is $17.5 \mathrm{kcal} / \mathrm{mol}$. This value is comparable to the value of the Lewis acid catalyzed degradation of $\operatorname{PVAc}^{8}(14.2 \mathrm{kcal} / \mathrm{mol})$ but is much lower than that reported for the thermal noncatalytic degradation of PVAc. ${ }^{4}$ This indicates that the degradation of PVAc by PTS may be similar to the degradation by

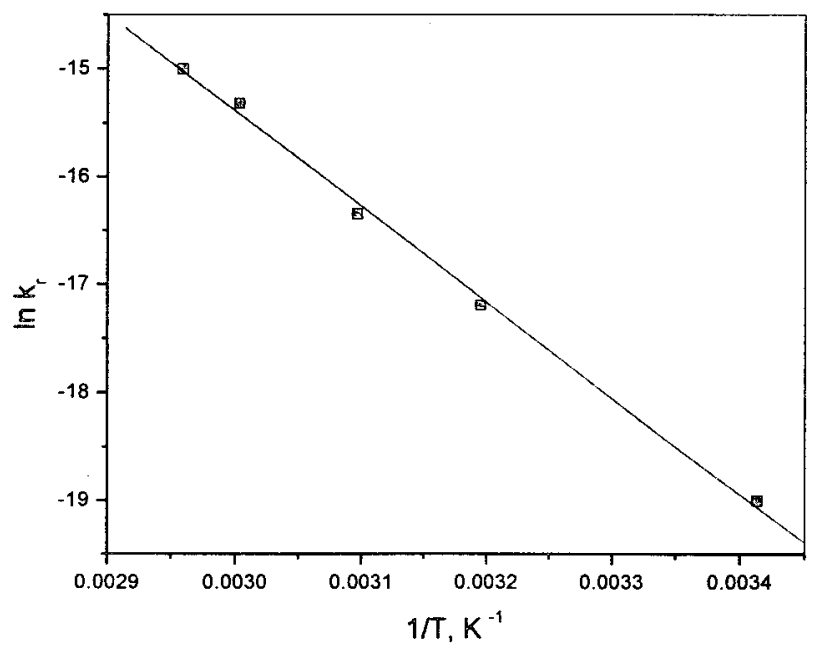

Figure 4 Arrhenius plot used to determine the activation energy for the acid-catalyzed degradation of PVAc. 
other Lewis acids by an ionic mechanism and may involve more than one rate-controlling step.

\section{CONCLUSIONS}

The degradation of PVAc has been investigated in toluene solutions by two different catalysts: an enzyme and PTS. Although the enzymatic degradation of the polymer results in specific products, the acidcatalyzed degradation of the polymer results in random products. The variation of the rate coefficients with the temperature for the enzymatic degradation indicates an optimum temperature of $55^{\circ} \mathrm{C}$. The variation of the rate coefficients with the temperature for the acid-catalyzed degradation follows an Arrhenius relationship with an activation energy of $16.5 \mathrm{kcal} /$ mol. This investigation shows that a cheap and commercial enzyme can be used for polymer degradation and that PVAc is degradable by enzymes.

\section{References}

1. Bikiaris, D.; Prinos, J.; Panayiotou, C. Polym Degrad Stab 1997, $56,1$.

2. Eling, B.; Gogolewski, S.; Pennings, A. Polymer 1982, 23, 1587.

3. Kulkarni, S.; McCarthy, S. P.; Gross, R. A. Annu Tech Conf 1995, $48,3232$.

4. Madras, G.; Chattopadhyay, S. J Appl Polym Sci 2001, 81, 1996.

5. Wang, M.; Smith, J. M.; McCoy, B. J. AIChE J 1995, 41, 1521.

6. Srivastava, S.; Modak, J. M.; Madras, G. Ind Eng Chem Res 2002, 41, 1940.

7. Srivastava, S.; Madras, G. J Chem Technol Biotechnol 2001, 76, 890.

8. Chattopadhyay, S.; Madras, G. Polym Degrad Stab 2001, 73, 83. 